\title{
DESAFÍOS DE LA POLÍTICA SOCIAL DE BRASIL: ¿CÓMO MANTENER EL CRECIMIENTO ECONÓMICO CON INCLUSIÓN SOCIAL? 1
}

\section{CHALLENGES OF SOCIAL POLICY IN BRAZIL: HOW TO MAINTAIN THE SOCIAL INCLUSION WITH ECONOMIC GROWTH?}

\section{Lena Lavinas*}

\begin{abstract}
RESUMEN
El presente artículo tiene el objetivo de mostrar los cambios registrados recientemente en el marco de la política macroeconómica de Brasil y los efectos de la misma en la política social, en particular en lo que concierne a la reducción de la pobreza. A partir de 2003, surge un nuevo patrón de crecimiento económico, con una expansión significativa del mercado doméstico desde el aumento del ingreso y del consumo de los segmentos medios de bajo poder adquisitivo. Dicho crecimiento tiene un impacto importante sobre la reducción de la pobreza y la desigualdad. El artículo esboza los desafíos que persisten en lo que concierne a la promoción de la equidad e inclusión social. Una tarea pendiente es la reforma fiscal que debería apalancar el cambio del perfil fuertemente recesivo de la financiación de la política social, tema que no se incluye en la agenda.
\end{abstract}

PALABRAS CLAVE: POLÍTICA SOCIAL * CRECIMIENTO ECONÓMICO * POBREZA * EQUIDAD

1 En colaboración con Camila Ferraz, Máster en Economía, Instituto de Economía de la Universidad Federal de Rio de Janeiro.
Instituto de Economía de la Universidad Federal de Río de Janeiro, Brasil.

lenalavinas@gmail.com 


\section{ABSTRACT ${ }^{\prime}$}

This article aims to present recent changes in the macroeconomic policy framework in Brazil and its effects on social policy, particularly with regard to poverty reduction. From 2003 onwards, a new pattern of economic growth prevails, reducing poverty and inequality on the grounds of domestic market expansion and low income groups consumption. This paper also outlines the remaining challenges for achieving social equity and inclusion, highlighting a major issue in the pending agenda: a progressive tax reform in order to leverage the financing of universal social policies.

KEYWORDS: SOCIAL POLICY * ECONOMIC GROWTH * POVERTY * EQUITY

\section{INTRODUCCIÓN}

La primera década del siglo XXI llega al Brasil de la mano de un clima de optimismo, consecuencia en gran medida, de la recuperación del crecimiento económico no solo a niveles más elevados sino dentro de una dinámica más sólida que la observada en los años 90 . Una de las grandes frustraciones nacionales con el mito de "Brasil, el país del futuro" desde el retorno de la democracia - las primeras elecciones libres para presidente de la República se llevaron a cabo en 1989-y desde la conquista de la estabilidad económica, yace en el hecho de que los mecanismos de movilidad social ascendente son casi inexistentes.

El recuadro 1 relata la sucesión de gobiernos que se desempeñaron en el poder ejecutivo federal brasileño desde el retorno de la democracia. El cambio de patrón de crecimiento junto a una vivencia democrática plena, se registra únicamente en la gestión más reciente del gobierno Lula (2003-2010).

\section{RECUADRO 1}

$\diamond \quad$ Fernando Collor (1990-1992): inicio del proceso de desestatización y privatización de la economía, con énfasis en un proceso de apertura económica indiscriminada.

$\diamond \quad$ Itamar Franco (1992-1994): lanzamiento del plan de estabilización que logra reducir las altas tasas de inflación.

$\diamond \quad$ Fernando Henrique Cardoso (1995-1998/1999-2002): consolidación de la estabilidad económica, ampliación del proceso de privatización, adopción de principios neoliberales, asistencia social fuera del marco del Sistema de Seguridad Social.

$\diamond \quad$ Luiz Inácio Lula de la Silva (2003-2006/2007-2010): recuperación del crecimiento a niveles más elevados y de forma sostenida, se mantiene la estabilidad, ampliación del mercado doméstico, saneamiento de las cuentas del país; reducción de la pobreza y de la desigualdad, creación de 13 millones de empleos formales, creación de un programa de transferencias condicionadas a gran escala dentro del ámbito del Sistema de Seguridad Social.

El cambio del patrón de crecimiento se debe al hecho de que el mismo tiene lugar a partir de la inclusión social y económica (más puestos de trabajo) de grupos sociales, que en períodos anteriores de fuerte crecimiento no representaban el motor propulsor de la demanda, conjuntamente con la expansión del crédito como fomento del desarrollo del mercado doméstico (nuevas líneas de financiación) y una mayor protección social, dado que 
la gran mayoría de los nuevos puestos de trabajo creados son formales. Asimismo, el nuevo patrón de crecimiento tiene una dinámica bastante más desconcentrada en todo territorio nacional y reduce las desigualdades regionales en términos de bienestar y desarrollo.

\section{LA MACROECONOMÍA DEL DESARROLLO}

Brasil registra un aumento en las tasas de crecimiento promedio del PIB en los cuadrienios seleccionados, con un aumento significativo en la gestión del Presidente Lula (de 2003 a 2010), como indica el gráfico 1.

PIB: TASA REAL DE CRECIMIENTO ANUAL (1995-2010)

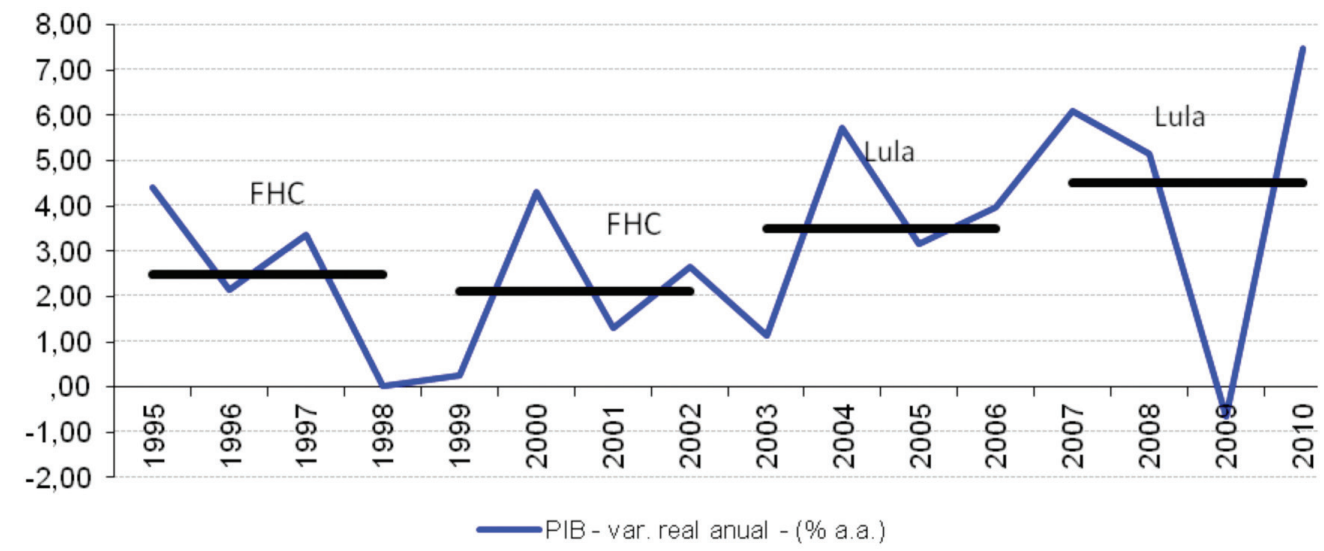

Fuente: IBGE/CCN.

En 2010, Brasil registra un crecimiento del 7,5\% del PIB, una evolución en recuperación de la actividad económica sin igual desde 1986.

Asimismo, durante el periodo anteriormente mencionado, se registra una caída abrupta de la tasa de inflación a niveles aceptables $(5,9 \%$ en 2010) y una leve, aunque constante reducción del índice de Gini que mide el nivel de desigualdad, con una reducción en el valor del índice de 0,604 en 1993, al nivel más bajo registrado en la serie ${ }^{2}$ de la encuesta del PNAD (Encuesta Nacional de Hogares-IBGE) a 0,540 en 2010 (tabla 1). Sin duda, el aumento de la oferta de empleo fue un aporte significativo para dicho resultado.

En este sentido, uno de los factores más alentadores del optimismo de los brasileños fue la creación de nuevos puestos de trabajo en el sector formal de la economía, con la reducción resultante en las relaciones de trabajo informales. Durante los ocho años del gobierno Lula, de enero de 2003 a diciembre de 2010, el

2 Serie anual de unidades residenciales con inicio en 1976, siempre referente al mes de septiembre.
TABLA 1

TASA DE INFLACIÓN (IPCA) E INDICE DE GINI

\begin{tabular}{ccc}
\hline AÑO & IPCA & GINI \\
\hline 1993 & $24,77 \%$ & 0,604 \\
2003 & $9,30 \%$ & 0,583 \\
2010 & $5,91 \%$ & 0,54 \\
\hline
\end{tabular}

Fuente: Ipeadata

saldo neto ${ }^{3}$ de empleos formales de acuerdo con CAGED $^{4}$ es de 11300 000, cifra record. El saldo neto de empleos formales solamente en el año 2010, es de 2300000 .

3 Considera la diferencia entre contrataciones y despidos de los trabajadores adheridos a la CLT (Consolidación de Leyes Laborales de Brasil, con vínculo formal $y$ laboral).

$4 \quad$ CAGED por las siglas en portugués - Registro General de Empleados y Desocupados, Ministerio del Trabajo, Brasil. 
El gráfico 2, muestra la evolución del empleo formal entre la gestión de FHC
(Fernando Henrique Cardoso) y la de Lula.

GRÁFICO 2

VALORES PROMEDIO DE NUEVOS PUESTOS DE TRABAJO FORMALES POR CUADRIENIO

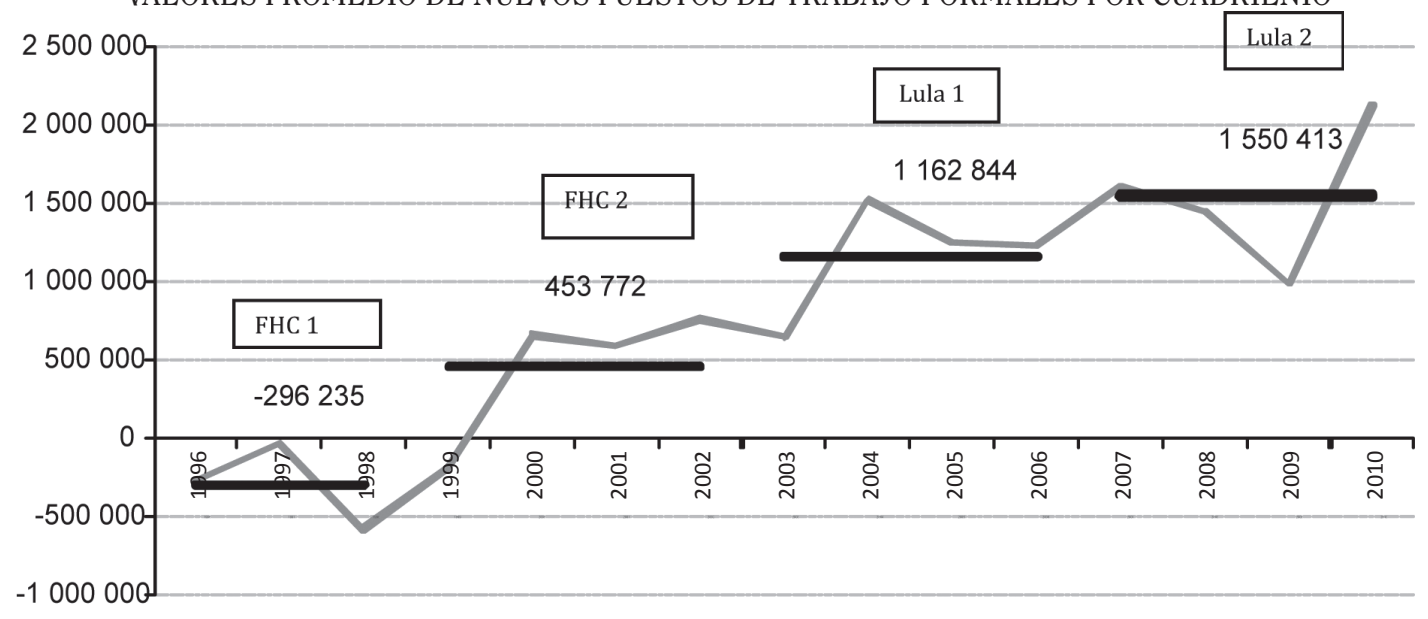

Fuente: CAGED/MTE.

Saldo del Empleo Formal

Vale mencionar que durante parte del primer mandato de FHC se eliminaron casi 300000 puestos de trabajo formales, con la consiguiente disminución del nivel de protección para el trabajador y su familia. El segundo mandato de FHC invierte dicha tendencia $y$ registra una recuperación significativa, aunque mucho menor que el aumento abrupto que se registra desde el primer mandato de Lula, cuando el saldo de empleos formales es dos veces y medio más elevado que el que consta en el cuadrienio anterior del antecesor en el cargo. El aspecto novedoso, por lo tanto, no reside solo en la creación masiva de empleos, sino en el hecho que son empleos de calidad, que garantizan la protección social del trabajo mediante aportes obligatorios al sistema previsional.

El Censo Demográfico de 2010, publicado recientemente, confirma que la tasa de formalización del empleo en Brasil llegó al 62,5\%, un nivel casi sin precedentes en el país. Este acontecimiento se debe al hecho de que el empleo formal registra un aumento de aproximadamente un 45\% del 2001 al 2010, a la vez que el empleo informal registra un crecimiento bastante menos expresivo, del $9 \%$ en el mismo período. El empleo formal se traduce en aportes al seguro social y en protección para el trabajador o su dependiente, ante el acontecimiento de una situación de riesgo.

El gráfico 3, muestra una tasa de desocupación media con tendencia a la baja durante el período y un valor de 6,7\% en el 2010.

Conjuntamente con el aumento de la oferta de empleo, el salario medio real inicia una tendencia hacia la recuperación en la década del 2000, aunque a un ritmo menor si es comparado con la creación de nuevos puestos de trabajo. Sin embargo, la masa salarial registra un aumento real del 40\% desde el 2003 al 2010.

El salario mínimo fue otro factor determinante para el aumento de la masa salarial: la recuperación del valor real del piso salarial nacional tendrá impacto, asimismo, en la reducción de las desigualdades regionales. El gráfico 4 registra la evolución de la recuperación del valor real del salario mínimo, con ganancias reales significativas, en especial a partir de 2005. En enero de 2010, el salario recupera el valor real que registraba en marzo de 1968, o sea que dicha recuperación llevó 40 años. 


\section{GRÁFICO 3}

VARIACIÓN DE LA TASA DE DESOCUPACIÓN DE 2003 A 2010

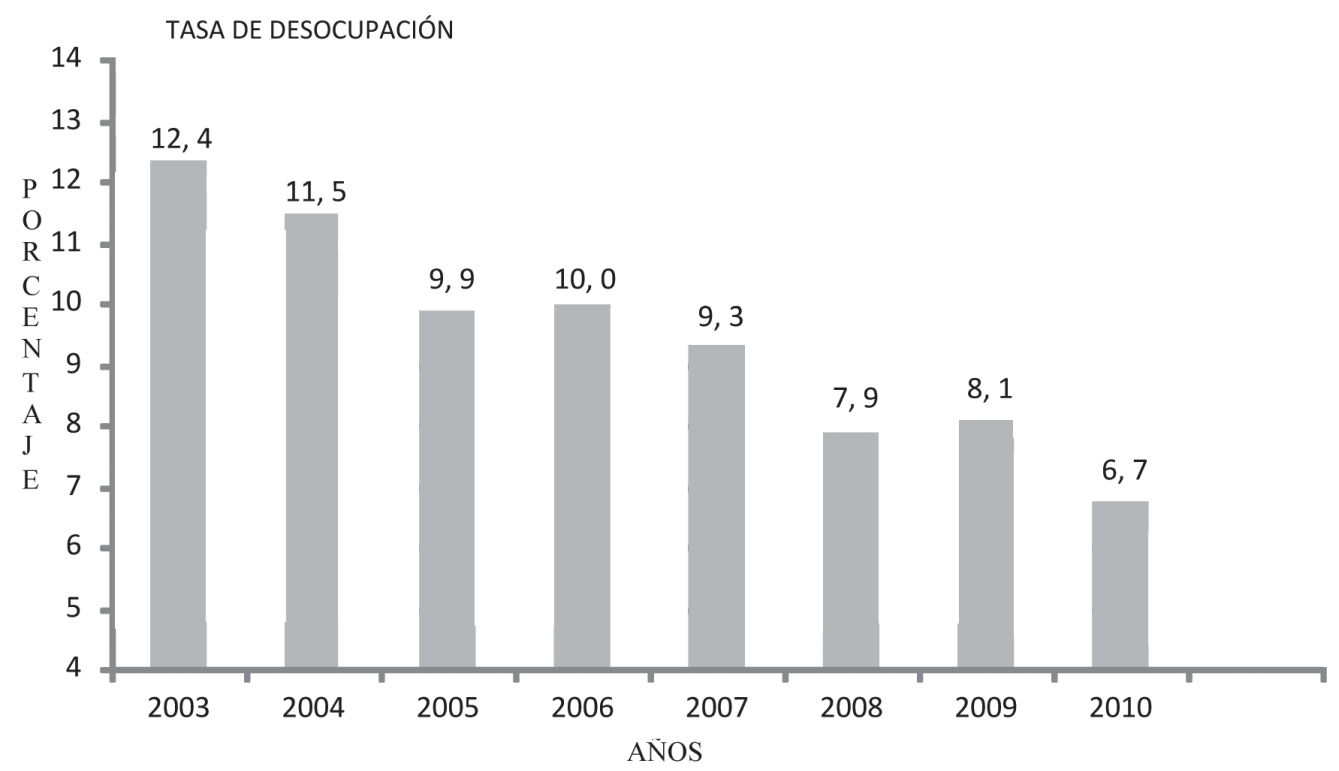

Fuente: IBGE.

GRÁFICO 4

EVOLUCIÓN DE LA RECUPERACIÓN DEL VALOR REAL DEL SALARIO MÍNIMO

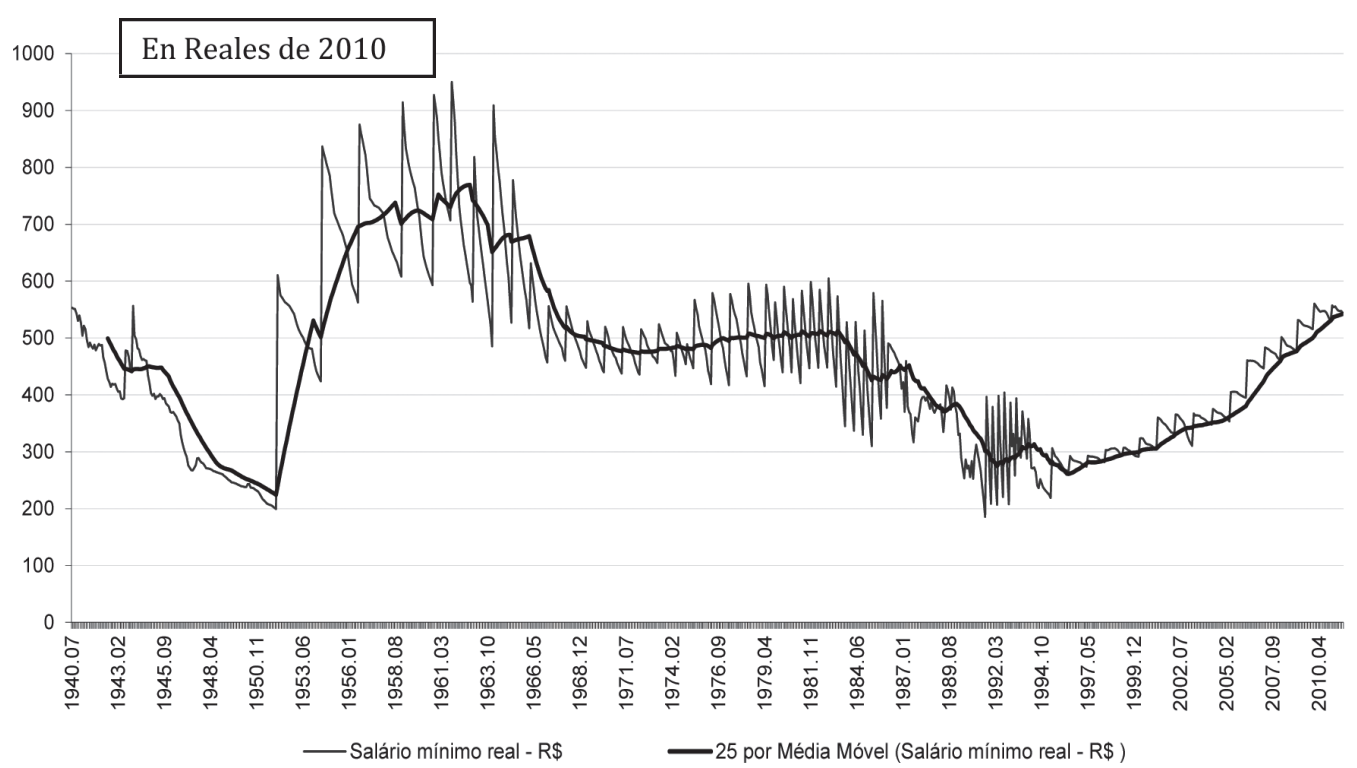

Fuente: IPEADATA. 
Al mismo tiempo, vale recordar que la masa salarial aun representa una pequeña parte del PIB. De acuerdo al Directorio de Estudios Macroeconómicos del Instituto IPEA (2010), la misma correspondía al 35,2\% del PIB en 1995, cayó al 30,8\% en 2004 y según proyecciones, registró un crecimiento en los años siguientes, sin recuperar por completo el peso relativo observado en 1995. Este valor sería del 34,1\% del PIB en 2009. Dicho dato es sumamente significativo para comprender que a pesar de una leve desconcentración salarial que propició una disminución del Índice de Gini en años recientes, Brasil sigue siendo un país de alta concentración de ingresos.

Finalmente, cabe destacar otro factor relevante (colateral), que ha registrado en los últimos años una evolución ascendente bastante positiva debido a la fuerza del aumento del empleo formal: la oferta del crédito, en particular del crédito personal, que estuvo reprimida en décadas anteriores debido a la coyuntura de bajo crecimiento. A pesar de que la tasa de interés se ha mantenido a niveles extremadamente altos durante la década, se registró un aumento en los plazos de pago que conjuntamente con el aumento de ingresos propiciado por la creciente oferta de puestos de trabajo, brindó la posibilidad de que más personas pudiesen solicitar préstamos en el mercado financiero.

El gráfico 5 muestra que el crédito pasa de $25 \%$ a $44,9 \%$ como proporción del PIB de 2004 a 2010. En valores absolutos, el stock de crédito referencial para la tasa de interés divulgado por el Banco Central, presentó un alza de $\mathrm{R} \$ 317,6$ mil millones en enero de 2004 a R\$ 871,7 mil millones en noviembre de 2010, registrando un aumento del $174 \%$ en el período.

\section{GRÁFICO 5 \\ EVOLUCIÓN DEL CRÉDITO COMO PROPORCIÓN DEL PIB (VARIACIÓN ANUAL)}

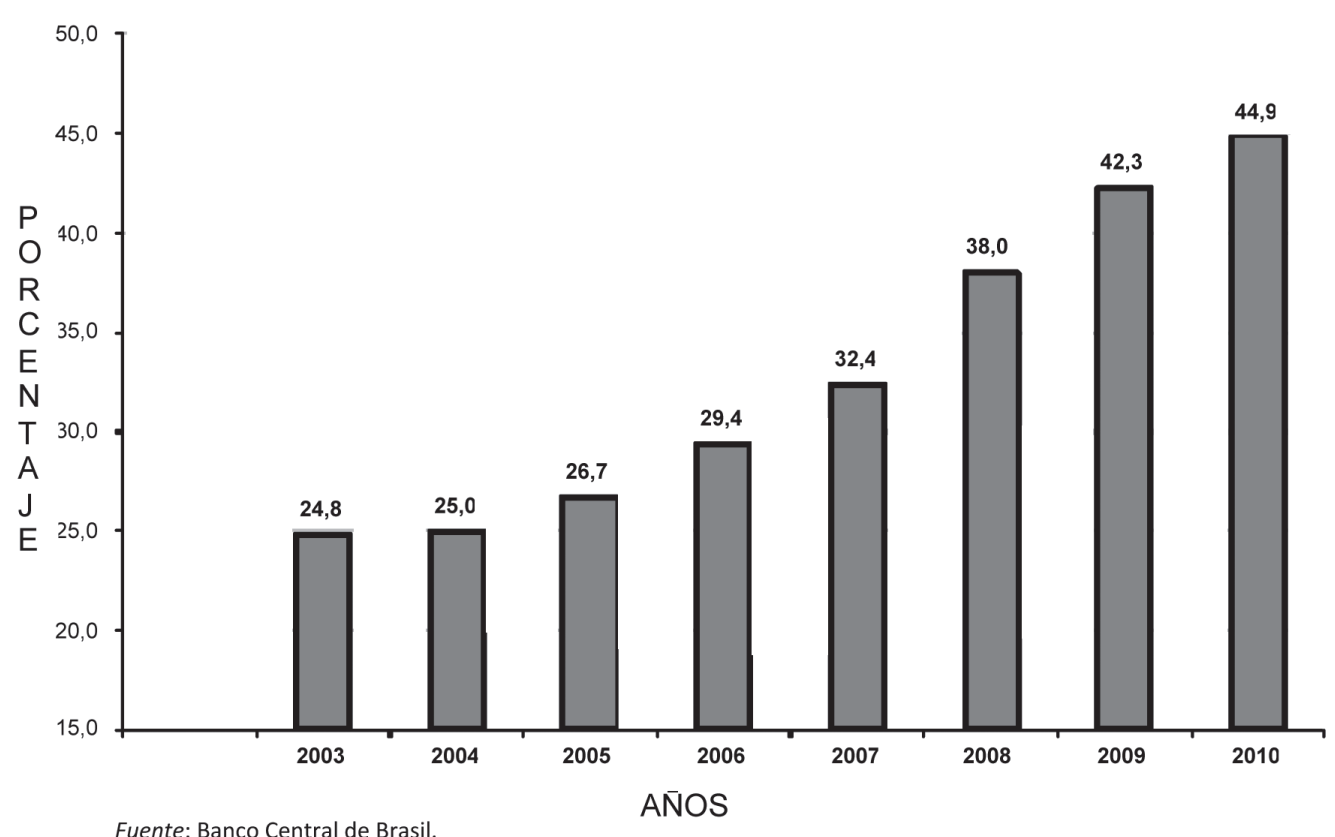

El crédito otorgado a personas físicas registró un aumento del 160\% de enero de 2004 a febrero de 2010. Sin lugar a dudas, la tendencia más acentuada y clara de la reciente expansión del crédito personal es reflejo de los cambios en el Sistema Financiero Nacional, los cuales permitieron a las familias un mayor acceso a las modalidades de financiación existentes, pero también una mayor capacidad de endeudamiento de las mismas. La fuerte tendencia 
en alza es la consecuencia de una de las innovaciones institucionales de 2003, la creación del crédito en nómina que brinda la posibilidad de reducir las tasas de interés y de morosidad.

La Ley 10820 del 17 de diciembre de 2003 en la gestión del gobierno de Lula, autoriza el otorgamiento de crédito en nómina a los trabajadores adheridos a la CLT. Posteriormente, la Ley 10953 de septiembre de 2004, enmienda la anterior extendiendo el crédito en nómina a jubilados y pensionados del sistema de previsión social de Brasil, denominado INSS por sus siglas en portugués. Por lo tanto, la creación del crédito en nómina favorece inicialmente a los empleados públicos y a los trabajadores adheridos a la CLT. El denominado "Préstamo Personal con Deducción en Nómina de Pago" se difundió rápidamente en la banca minorista de todo el país en manos de los trabajadores con un empleo fijo, estable y prácticamente sin riesgos, además de los empleados públicos contratados de conformidad con el marco legal brasileño. Al año siguiente, se instituye dicha modalidad para jubilados $y$ pensionados dentro del marco del Inss.

De acuerdo con Lavinas, Ferraz y Veiga (2012), el valor de las cuotas de este tipo de préstamo se debita directamente del sueldo y el Consejo Nacional de Previsión Social fija la tasas de interés que cobran las financieras, bancos o mutuales de crédito. De conformidad con la Ley y el Decreto 4840/03, las cuotas del préstamo en nómina no pueden superar el 30\% del valor del salario o remuneración que se utiliza como garantía. Este tipo de préstamo se encuentra disponible únicamente para los titulares de beneficios otorgados por el INSS, a pesar de que en la práctica, los titulares del Beneficio de Prestación Continua (BPC), administrado y otorgado por el INSS, no pueden acceder a la línea de crédito especial. Por su parte, existe un punto de inflexión en el acceso al préstamo en nómina: el mismo se aplica a las remuneraciones contributivas, pero no se aplica a los beneficiarios de pensiones no contributivas, como el Beneficio de Prestación Continua (BPC $)^{5}$. Vale

$5 \quad$ El Beneficio de Prestación Continua, consagrado en la Constitución de 1988 e implementado a partir de 1993, otorga un beneficio del valor de un salario mínimo a los deficientes (discapacitados) destacar que no incide el valor del beneficio el valor del BPC es igual al piso del beneficio previsional, a saber, un salario mínimo vigentesino el status del beneficiario, contribuyente o trabajador. Los que gozan de status de no contribuyente no pueden optar por el crédito. Una de las conclusiones del estudio de Lavinas, Ferraz y Veiga (2010) es que el crecimiento de la oferta de crédito no logra solucionar los problemas de acceso de los segmentos más pobres, con llegada solo al crédito de la banca minorista para la compra de bienes de consumo durables $y$ bienes-salario, de importante efecto multiplicador en la sostenibilidad de la actividad económica y expansión del mercado doméstico, en especial en 2009, año de la grave recesión desencadenada por la crisis internacional. La denominada "inclusión financiera" de los más pobres sigue siendo una asignatura pendiente.

Dentro del ámbito del crédito personal, el crédito en nómina representa el 46\% del total del mismo en 2010 comparado al 25\% en 2004. El volumen de los créditos en nómina se duplica con creces anualmente. A su vez, la innovación tuvo éxito gracias al quehacer de la banca pública dentro del ámbito de una política pública de ampliación del acceso a la financiación, como consta en el gráfico 6.

Se han presentado algunos elementos que explican el comportamiento de los índices de pobreza y de medición de la desigualdad en los últimos años, especialmente, a partir del segundo mandato del presidente Lula. A continuación, se considerarán los aspectos relativos al marco macroeconómico de recuperación del empleo y del valor real del salario mínimo, en una coyuntura de recuperación sostenida del crecimiento económico, para interpretar lo que sucede en términos de la inclusión social y la mejoría de los niveles agudos de destitución vigentes en el país.

\footnotetext{
o adultos mayores de más de 65 años de edad, a los miembros de una familia con ingreso familiar per cápita por mes igual o inferior a $1 / 4$ del salario mínimo vigente.
} 


\section{GRÁFICO 6 \\ CRÉDITO EN NÓMINA (\% SOBRE EL TOTAL DE CRÉDITO EN NÓMINA) \\ DE ORIGEN PÚBLICO Y PRIVADO}

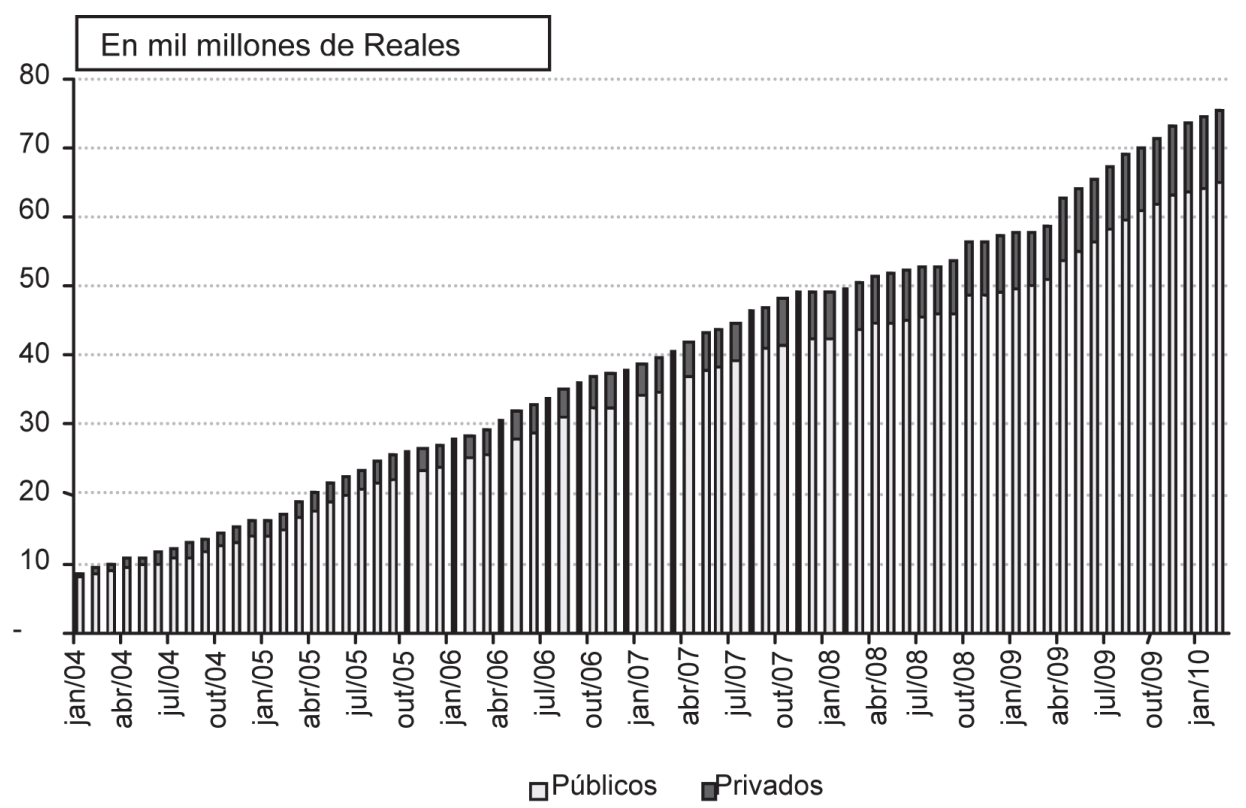

Fuente: Banco Central de Brasil.

Se debe considerar el contexto macroeconómico y la expansión del empleo $y$ del consumo doméstico, este último también fomentado por el aumento de la oferta de nuevos productos de crédito, para dimensionar las variaciones en la magnitud de los índices de pobreza y desigualdad del Brasil del nuevo milenio. Sin embargo, sin la vigencia de un nuevo marco regulatorio para las relaciones sociales, con base en la ampliación de los derechos de ciudadanía de los segmentos anteriormente excluidos y consagrado en la Constitución de 1988, habría sido casi imposible que el contexto macroeconómico por sí solo, fuera el propulsor de un proceso significativo de reducción de la pobreza y de la desigualdad, característica singular de la nueva etapa de desarrollo económico con inclusión social.

\section{POBREZA: EVOLUCIÓN RECIENTE}

Los datos de la tabla 2 recogidos de la Encuesta Nacional de Hogares, encuesta del PNAD del IBGE, revelan una reducción sostenida de los índices de pobreza e indigencia registrados en Brasil, del 2001 al 2009, fecha de la última encuesta disponible por el IBGE ${ }^{6}$.

Las líneas de pobreza e indigencia utilizadas por el Programa Bolsa Familia se utilizaron como corte de ingresos para la identificación de los grupos, debido al hecho de que Brasil no cuenta con una línea de pobreza oficial. Se define como pobres a las personas que viven en familias con un ingreso familiar per cápita igual o inferior a $R \$ 140,00$ por mes, en el año 2010 . Se define como indigentes o extremadamente pobres a las personas que viven con un ingreso familiar per cápita igual o inferior a $\mathrm{R} \$ 70,00$. Para los efectos del presente artículo, las categorías pobre e indigente se consideran por separado.

A partir del análisis de la tabla 2, se identifica una reducción bastante significativa de los índices de pobreza extrema, que de $15,6 \%$ en 2001 caen a $5,4 \%$ en 2009 . Es decir, que en ocho años, $2 / 3$ de los brasileños abandonaron el segmento de miserables. En valores absolutos esta cantidad representa a 16800000 personas.

$6 \quad$ El Censo Demográfico de 2010 aun se encuentra en la fase crítica y de limpieza de universo. No se han publicado los datos. 
TABLA 2

PROPORCIÓN DE POBRES E INDIGENTES EN LA POBLACIÓN BRASILEÑA

\begin{tabular}{ccc}
\hline AÑO & POBRES (\%) & INDIGENTES (\%) \\
\hline 2001 & 33,3 & 15,6 \\
2004 & 22,1 & 8,5 \\
2007 & 19,2 & 8,2 \\
2008 & 15,5 & 6,5 \\
2009 & 10,1 & 5,4 \\
\hline
\end{tabular}

Fuente: PNAD, IBGE, años citados con base en el ingreso familiar per cápita.

Dentro del ámbito de la pobreza, la disminución es igualmente significativa en términos relativos, dado que la proporción de pobres cae del 33\% de la populación en 2001, al 10,1\% en 2010. Aproximadamente, 58000000 vivían por debajo de la línea de pobreza per cápita del Programa Bolsa Familia en 2001. Dicho valor cae a cerca de 19000000 de personas en 2010. Cálculos estimativos indican que la suma de indigentes y pobres totaliza 29000000 de personas en el 2010, o sea, aproximadamente el $15 \%$ de la población. Esta cifra aun es un contingente muy significativo de personas.

La tabla 3 desglosa los ingresos familiares per cápita de acuerdo a las tres fuentes registradas en la encuesta del PNAD, a saber, ingresos del trabajo, ingresos de jubilaciones $y$ pensiones e ingresos de otras fuentes que componen el total de los ingresos monetarios familiares. Las denominadas "otras fuentes" se componen de ingresos diversos como las define el propio IBGE, tales como pago de alquileres, cobro de dividendos y también, en el caso de los más pobres, transferencias condicionadas de ingresos, si el titular es beneficiario de algún programa o política de alguna instancia de gobierno. En el caso de la población ubicada en las primeras decenas de la distribución, la clasificación "otras fuentes" se utiliza como sustituto del beneficio del Programa Bolsa Familia, BPC (Beneficio de Prestación Continua) u otro beneficio asistencial equivalente que calificara a una familia como institucionalmente pobre.
Para los fines del presente estudio, vale recordar que la línea de pobreza adoptada es la vigente dentro del marco del Programa Bolsa Familia en cada uno de los años seleccionados. En el caso del año 2001, antes de la creación del Programa Bolsa Familia, se utiliza el valor deflacionado del 2003 para que el mismo sea constante y comparable. El presente artículo adopta los mismos criterios de comparación para la línea de indigencia. De este modo, todos los valores por año son comparables y consistentes.

Si se consideran los datos de la tabla 3 para todo Brasil, los ingresos del trabajo del $45,4 \%$ de los brasileños se ubicaban en el segmento de la pobreza en 2001. Este porcentaje cae a $30 \%$ en 2008 , o sea que en siete años, 21000000 de personas dejaron de ser pobres, básicamente gracias a la recuperación sostenible del crecimiento a partir de 2003 (auge en inversiones, a niveles que superan en mucho las tasas de crecimiento del PIB) y a la recuperación del salario mínimo (con reajustes por encima de la inflación, con aumentos reales). Los dos puntos anteriores conjuntamente promovieron nuevas oportunidades de empleo con crecimiento real de los ingresos del trabajo. A pesar de este reconocimiento, si se consideran exclusivamente los ingresos del trabajo, el número de brasileños que viven en la pobreza llega a los 57000 000, valor prácticamente idéntico al registrado en 2001, si se toman en consideración todas las fuentes de ingresos. Desde esta perspectiva, se puede afirmar que en el año 2008, el trabajo aun no es suficiente como para brindar una vida digna a un número significativo de brasileños (1/3 de la población).

Por su lado, gracias a las jubilaciones y pensiones, ya sea dentro del marco del Régimen General de Previsión Social (RGPS) o del Régimen Público (RPPS), el número absoluto y relativo de pobres registra una disminución sustancial del 12\% (que equivale a 22000000 de personas menos) y si se consideran como fuentes de ingresos al trabajo y beneficios contributivos, el índice de pobreza llega al 18,7\%. Esto significa que el sistema de jubilaciones no es regresivo, al beneficiar solo a los acogidos por el beneficio del seguro social, contribuyentes del sector formal de la 
TABLA 3

INGRESOS FAMILIARES PER CÁPITA SEGÚN FUENTES REGISTRADAS EN ENCUESTA DEL PNAD

\begin{tabular}{lcccc}
\hline \multicolumn{5}{c}{ NÚMERO DE POBRES } \\
\hline POBRES & 2008 & 2007 & 2004 & 2001 \\
\hline Ingresos del Trabajo + Pensiones + Cash Transfers & 29497278 & 36322306 & 40395722 & 57246811 \\
Ingresos del Trabajo + Pensiones Contributivas & 35466179 & 41244408 & 45453053 & 59410723 \\
Ingresos del Trabajo únicamente & 57101997 & 62961022 & 66598844 & 78058032 \\
\hline
\end{tabular}

INDIGENTES

\begin{tabular}{lllll}
\hline Ingresos del Trabajo + Pensiones + Cash Transfers & 12350063 & 15428976 & 15590548 & 26865279 \\
Ingresos del Trabajo + Pensiones Contributivas & 17773725 & 20592390 & 20698258 & 29143176 \\
Ingresos del Trabajo únicamente & 36528070 & 39468703 & 38893286 & 47012898 \\
\hline
\end{tabular}

PROPORCIÓN DE POBRES (\%)

\begin{tabular}{lcccr}
\hline & 2008 & 2007 & 2004 & 2001 \\
\hline POBRES & & & & \\
\hline Ingresos del Trabajo + Pensiones + Cash Transfers & 15,5 & 19,2 & 22,1 & 33,3 \\
Ingresos del Trabajo + Pensiones Contributivas & 18,7 & 21,8 & 24,9 & 34,6 \\
Ingresos del Trabajo únicamente & 30,1 & 33,3 & 36,5 & 45,4 \\
\hline
\end{tabular}

INDIGENTES

\begin{tabular}{lrrrr}
\hline Ingresos del Trabajo + Pensiones + Cash Transfers & 6,5 & 8,2 & 8,5 & 15,6 \\
Ingresos del Trabajo + Pensiones Contributivas & 9,4 & 10,9 & 11,3 & 17,0 \\
Ingresos del Trabajo únicamente & 10,2 & 20,9 & 21,3 & 27,4 \\
\hline
\end{tabular}

Fuente: IBGE, PNAD, 2001, 2004, 2007 y 2008.

economía. De esta manera, toman importancia las jubilaciones rurales para hombres y mujeres, de igual valor y sin aportes previos, conjuntamente con el hecho de que el salario mínimo está vinculado al piso previsional, ambas conquistas consagradas en la Constitución de 1988. Por otro lado, la regla de reajuste del salario mínimo nacional sufrió modificaciones recientes que incorporan ganancias reales importantes cuando se registra crecimiento económico. El salario mínimo se reajusta anualmente en enero de acuerdo a la inflación del año anterior y con la tasa de crecimiento del PIB de dos años anteriores. El reajuste del salario mínimo en 2012 , por ejemplo, debe ser aproximadamente del $14 \%$, o sea $6,5 \%$ relativos a la expectativa de inflación acumulada en 2011 sumado a 7,5\% referentes al aumento real del PIB registrado en 2010.

Las otras transferencias fiscales no contributivas o asistenciales reducen el segmento de pobres un 3\% más, a 29400000 personas o el $15,5 \%$ de la población. Esto significa que aproximadamente 7500000 de familias viven por debajo de la línea de pobreza de $\mathrm{R} \$ 140,00$ per cápita.

En resumen, se observa que durante la mayor parte de la década pasada, el número de pobres disminuye de 57200000 personas a menos de 30000 000, si se considera una línea de pobreza extremadamente baja que corresponde a $\mathrm{R} \$$ 4,5 per cápita/día aproximadamente, o 
USD 2,5 per cápita/día, muy parecida al patrón del Banco Mundial. En las grandes ciudades brasileñas no se pueden tomar dos colectivos en un día con ese valor.

En lo relativo a la indigencia, sin duda, el efecto acumulativo del crecimiento con elevación de los ingresos del trabajo, más empleo $y$ una mayor cobertura de los programas asistenciales de transferencia de ingresos condicionados es mucho más efectivo. En cuanto a la pobreza, todas las transferencias de ingresos acumuladas (contributivas y no contributivas) reducen el índice de pobreza a la mitad, con una disminución del 30\% al 15\%. Para la indigencia o pobreza extrema, dicho efecto es más amplio: la proporción de indigentes se reduce de $19,2 \%$ a $6,5 \%$, o sea, una disminución de 2/3 (de 36000000 de personas a 12000000 o 3600000 familias indigentes en el mismo año). La indigencia monetaria registró una reducción más efectiva que la pobreza.

Con todo, cabe recordar que la línea de indigencia es actualmente de R\$2,3 per cápital día (o USD 1,4 per cápita/día). ¡Una línea casi indigente! Se puede suponer que, adoptada una línea de pobreza y de indigencia más elevada, que sea compatible con el costo de vida y la provisión de una serie de necesidades básicas, la proporción de pobres e indigentes sería mayor.

Se debe subrayar que no se ubicaron o se acogieron al beneficio asistencial del Programa Bolsa Familia tras las transferencias fiscales de 2009, a todas las personas que se encuentran por debajo de la línea de la pobreza, a pesar de que sean beneficiarias potenciales, dado que sus ingresos - criterio de selección casi exclusivo- cumplen con los requisitos establecidos por el importante programa centrado en transferencias condicionadas de ingresos a nivel nacional. De acuerdo a la encuesta del PNAD 2009, existen 5800000 unidades domésticas pobres con un ingreso per cápita por mes inferior a $\mathrm{R} \$ 140,00$, de los cuales 2200000 unidades domésticas declaran no contar con ningún tipo de beneficio asistencial público. Esta situación afecta aproximadamente a 9000000 de personas de un total estimado de 29000000 de pobres (casi $1 / 3$ ), una masa expresiva que no logra la inclusión y permanece al margen de la política social. Esta situación se explica por el hecho de que el Programa Bolsa Familia no es un derecho, sino un programa con un diseño y cobertura que obedece a restricciones presupuestarias y cuenta desde su origen, con un límite máximo de número de beneficiarios. Esta característica revela una de sus grandes debilidades: el Programa presenta gran ineficiencia horizontal, al generar inequidades entre los grupos más carenciados, como consecuencia de prácticas de focalización y del no reconocimiento del derecho a la ciudadanía por parte de la población destinataria de los programas asistenciales. La discriminación característica de la sociedad brasileña no es la excepción a la regla en ningún segmento social.

Si se considera también a la población vulnerable ${ }^{7}$, no solo el porcentaje se mantiene más o menos constante entre 2001 y 2009, es decir a 19\%, sino que la masa de población aumenta en términos absolutos en 1100000 personas aproximadamente, de 33500000 a 34600000 .

De esta manera, los grupos en la cola de la distribución totalizan 63500000 personas en 2009, lo que representa un contingente aun muy significativo de personas que viven en situación crítica o potencialmente de riesgo.

Por otro lado, la pobreza no monetaria no presenta una evolución tan favorable. Los indicadores que miden el bienestar de la población pobre a partir del nivel de acceso al saneamiento, la luz eléctrica y el abastecimiento de agua corriente, indican que se registraron avances en los últimos años pero que el pasivo remanente es aun significativo.

Los micro datos de la encuesta del PNAD indican que a pesar de la ampliación de la cobertura pública en lo que concierne al saneamiento de zonas urbanas, el 15\% de los hogares pobres urbanos no cuentan con abastecimiento de agua corriente y el $40 \%$ no cuenta con red cloacal adecuada en el 2009. El déficit

$7 \quad$ En este caso, las personas con ingreso familiar per cápita es equivalente a 1,8 de la línea de pobreza de $\mathrm{R} \$ 140,00$, de acuerdo a la metodología de la CEPAL. De esta manera, la línea de vulnerabilidad sería de aproximadamente $\mathrm{R} \$ 250,00$ per cápita por mes. 
del bienestar en las zonas rurales donde viven aproximadamente 30000000 de personas (de las cuales 10000000 son pobres) se agrava en gran medida: el 30\% de la población pobre del campo no cuenta con baño en sus hogares. Cerca del $7 \%$ vive sin luz eléctrica. La brecha entre la pobreza urbana y rural aun es profunda.

La inexistencia de una política para la vivienda durante más 50 años deja consecuencias dramáticas en las grandes metrópolis brasileñas: se generalizó la tugurización y las viviendas precarias como patrón prevalente en un escenario de gran degradación urbana y ambiental. El estudio de la Fundación João Pinheiro de 2010 (Ferraz, 2011), estima que el déficit habitacional es de 5500000 viviendas aproximadamente. Dentro de este contexto, las familias con ingresos de hasta tres salarios mínimos representan el 90\% del déficit habitacional. Actualmente, uno de los cuellos de botella más complejos para la promoción de una inclusión social decente generadora de oportunidades son las inversiones en infraestructura social $y$ en vivienda.

En este sentido, el perfil del gasto público debe cambiar, dado que el mismo enfoca la atención en las transferencias condicionadas de ingresos, $80 \%$ del gasto social, con impacto negativo en la prestación universal de un conjunto importante de servicios. Dicha situación reduce no solo las externalidades positivas, sino también las posibilidades de apalancamiento de los más pobres. El gasto público no propició un aumento del capital social por medio del aumento de la calidad y diversidad de la infraestructura urbana y social de las áreas más carenciadas donde se concentra la población más pobre. El entorno de la miseria es inhumano, el acceso a la salud universal es deficiente, la educación se ve perjudicada por la falta de una política a largo plazo con inversiones en la calidad y no solo orientada a la prestación de una cobertura universal.

La evolución positiva de la reducción de la pobreza se debe en gran medida a la institucionalidad del sistema de protección social brasileño fundado en 1988, cuando se promulga la nueva Constitución Ciudadana. Brasil cuenta en la actualidad, con un sistema integrado con presupuesto propio que se fundamenta en reglas y normas definidas $y$ de mayor uniformidad. Esta situación garantiza, por ejemplo, la igualdad de derechos entre hombres y mujeres de los segmentos urbanos y rurales en el ámbito de los derechos previsionales. Asimismo, se instituye la anteriormente inexistente asistencia social como derecho para todos los que se encuentran en situación de necesidad. La salud se convierte en un sistema universal público, no vinculado a la capacidad de aporte.

A continuación se presenta un resumen de la institucionalidad de la seguridad social brasileña:

$\diamond \quad$ Seguro social (diversos tipos de beneficios previsionales para los aportantes, de conformidad con reglas uniformes) CONTRIBUTIVO

$\diamond \quad$ Asistencia social para los carenciados (derecho a un mínimo social derivado de la comprobación del déficit de ingresos) NO CONTRIBUTIVO

$\diamond \quad$ Salud para todos, financiada por impuestos indirectos sobre el consumo - NO CONTRIBUTIVO

$\diamond \quad$ Seguro desempleo - CONTRIBUTIVO

Se crea dentro del marco de la asistencia social, un beneficio monetario denominado Beneficio de Prestación Continua (BPC) ${ }^{8}$, cuyo valor es de un salario mínimo, que se extiende a todo adulto mayor indigente (edad igual o superior a los 65 años $^{9}$ ) o portador de deficiencias

8 El BPC, el cual reemplaza al Ingreso Mensual Vitalicio (RMV por sus siglas en portugués), fue creado en 1974 como un beneficio previsional (por lo tanto, no solo para los que comprueban ser pobres), para los mayores de 70 años de edad o inválidos, definitivamente incapacitados para el trabajo, que en ambas situaciones, no se desempeñen en actividades remuneradas $y$ no tengan ingresos que superen el $60 \%$ del valor del salario mínimo. A partir de 1996, cuando entra en vigor el beneficio BPC, se interrumpe el programa RMV. La cobertura del mismo siempre fue menor y actualmente es residual, como consecuencia de su interrupción.

9 Inicialmente, solo podían acogerse al beneficio adultos mayores de más de 67 años de edad, pero la edad mínima disminuye posteriormente a 65 años. 
que viven en familias con un ingreso familiar per cápita inferior a $1 / 4$ del salario mínimo vigente. Es la primera vez que el Estado brasileño se compromete a reducir la pobreza extrema a través de una transferencia condicionada de ingresos para la población pobre efectivamente comprobada, adulta mayor o con deficiencias. Este derecho emana de la Ley Orgánica de Asistencia Social de 1993 y entra en vigor en 1996. El BPC se conforma como un componente integral de la Protección Social Básica dentro del marco del Sistema Único de Asistencia Social formalizado en 2004.

A partir de la gestión de gobierno de Lula, la asistencia social aumenta la participación en el presupuesto del país y amplía el goce de derechos a los pobres en general $y$ no solo a los adultos mayores o personas con deficiencias. Nace el Programa Bolsa Familia ${ }^{10}$, el programa de transferencias condicionadas de ingresos más importante de Brasil y Latinoamérica, eje fundamental de la política de lucha contra la pobreza del país. El Programa se reglamenta a fines del 2003 y la base inicial de beneficiarios estaba conformada por los actualmente denominados "Programas

10 El Programa Bolsa Familia forma parte de la estrategia Fome Zero (Hambre Cero) del actual gobierno cuyo objetivo consiste, por lo tanto, en la superación del hambre y la pobreza. Para dicho fin, se establecen en la articulación tres dimensiones esenciales:

$\diamond \quad$ Promoción del alivio inmediato de la pobreza, por medio de la transferencia directa de ingresos a la familia.

$\diamond \quad$ Refuerzo del goce de los derechos sociales básicos en el área de la Salud y Educación, por medio de la realización de transferencias condicionadas para que las familias logren quebrar el ciclo de pobreza entre generaciones.

$\diamond \quad$ Coordinación de programas complementarios cuyo objetivo es el desarrollo de las familias para que los beneficiarios del Programa Bolsa Familia logren superar la situación de vulnerabilidad y pobreza. Entre los ejemplos de programas complementarios se pueden mencionar: los programas de generación de trabajo e ingresos, de alfabetización de adultos, de tramitación del registro civil y demás documentos (MDS, 2010).
Remanentes" (Tarjeta Alimentación, Bolsa Alimentación, Bolsa Escuela y Ayuda Gas) ${ }^{11}$. El mismo tiene cobertura en todo el territorio nacional $y$ beneficia a aproximadamente 13000000 de familias o 45000000 de personas. Más de la mitad de los beneficiarios viven en las regiones norte $y$ noreste del país, regiones de menor desarrollo económico. El Programa cuenta con beneficios de valores distintos, que varían de un mínimo de $\mathrm{R} \$ 70,00$ a $\mathrm{R} \$ 275,00$ mensuales por familia.

Otra innovación institucional de la Seguridad Social fue equiparar los beneficios previsionales de los trabajadores rurales $y$ urbanos. El derecho de acogerse al beneficio de una jubilación o pensión de valor idéntico al piso previsional de los trabajadores urbanos, o sea, un salario mínimo, rige ahora para los trabajadores del campo y pequeños productores rurales que viven en régimen de explotación familiar, con la salvedad de que para los trabajadores rurales, el mismo no depende de un aporte previo integral. Las mujeres rurales que se desempeñan en la agricultura de subsistencia en régimen familiar, pueden jubilarse a partir de los 55 años de edad con derecho al cobro de un beneficio previsional del valor de un salario mínimo y los hombres, a partir de los 60 años de edad ${ }^{12}$.

En este sentido, varios autores ${ }^{13}$ han demostrado de qué manera los derechos mencionados anteriormente logran reducir el Índice de Gini a niveles extremadamente bajos y satisfactorios en el medio rural, el más bajo del

11 Con la excepción de la Tarjeta-Alimentación creada a principios de 2003 durante el gobierno de Lula, los demás "Programas Remanentes" se implementaron en la gestión de Fernando Henrique Cardoso, a partir de fines de los años 90. Posteriormente, los beneficiarios del PETI y del ProJoven Adolescente también fueron incorporados al primero, aunque manteniendo una estructura administrativa independiente.

12 Hasta 1988, la población rural, exclusivamente en calidad de jefes de la unidad de explotación familiar, cobraban el FUNRURAL, un beneficio previsional con un valor de medio salario mínimo y el cónyugue no cobraba nada.

13 Consultar sobre ese tema en particular, los artículos de Guilherme Delgado, en varias publicaciones del IPEA. 
país $(0,280)$, al margen de registrar una reducción significativa en las diferencias de género y rural/urbano.

\section{EL DESAFÍO DE TRANSFORMAR A LA SOCIEDAD BRASILEÑA EN UNA SOCIEDAD MENOS REGRESIVA}

Uno de los desafíos más importantes que tiene la sociedad brasileña en la construcción de una nación justa, equilibrada y que brinde a todos la posibilidad de valorizar por iniciativa propia, sus talentos y habilidades, en el disfrute de oportunidades reales, es reducir el nivel de regresividad que rige la financiación de la política social. Los recursos que garantizan el pago de los beneficios asistenciales y financian a la salud, se originan en los aportes indirectos realizados por la sociedad como un todo, inclusive por los pobres, cuya participación en la carga tributaria derivada del consumo representa del $25 \%$ al $40 \%$ de sus ingresos netos. Los estudios sobre la dinámica de la pobreza no consideran este punto, dado que el análisis parte de los ingresos brutos declarados.

La reforma fiscal pendiente desde el retorno a la democracia, no contempla en su estructura este punto de suma importancia. En lo relativo a exenciones $y$ deducciones fiscales del impuesto a las ganancias de personas físicas, la clase media y alta cuentan con un volumen de crédito tributario mucho más elevado que los gastos que representan las transferencias de ingresos condicionadas. El impuesto a las ganancias es un ejemplo de dicha situación. En este sentido, vale recordar que se aplican deducciones por dependientes, de valor correspondiente a un beneficio mensual que supera ampliamente el valor tope del Programa Bolsa Familia, por gastos de educación (limitadas) y sobre todo, deducciones ilimitadas por gastos en concepto de salud. El impuesto sobre el patrimonio es altamente regresivo e injusto, favorece la concentración de la riqueza y poder. El Índice de Gini de concentración latifundiaria en Brasil es del 0,840. Si el Índice de Gini, que se calcula a partir de los ingresos del trabajo, jubilaciones, pensiones y transferencias condicionadas de ingresos ${ }^{14}$, ha disminuido levemente de 0,594 en el 2000 a 0,538 en el 2010; el de la riqueza y de los activos muestran el mismo nivel de alta concentración en Brasil.

La crisis vuelve a colocar en pauta al rol fundamental del Estado en lo que concierne a la formulación e implementación de políticas anticíclicas. Los servicios sanitarios y la vivienda vuelven a las agendas como sectores estratégicos para enfrentar la crisis por medio de inversiones públicas, con generación masiva de puestos de trabajo y mantenimiento de la actividad económica. Sin embargo, una vez más, se impone la óptica cortoplacista de un conjunto de medidas del Programa, con metas bien definidas, sin considerar la dimensión compleja y a largo plazo de la formulación de una política. Llegó la hora de transformar programas en políticas.

La actual coyuntura hace un llamamiento no solo para evitar retrocesos, sino para garantizar avances reales hace mucho añorados, para consolidar un sistema de protección social incompleto, que puede mejorar su efectividad pero que evidencia su mayor imperfección en el hecho de ser aun excluyente y regresivo.

La nación brasileña acaba de asumir el compromiso de erradicar la miseria. Sin una política audaz para el enfrentamiento de las desigualdades y orientada hacia la desconcentración real de la riqueza, es poco probable que la agenda de crecimiento económico por si sola pueda promover más inclusión y progresividad.

Ha llegado la hora de las grandes reformas redistributivas.

\section{BIBIOGRAFÍA}

CAGED. Datos sobre empleo formal. Brasil: Registro General de Empleados y Desocupados, Ministerio del Trabajo, 2011.

Ferraz, Camila. "Crédito, exclusión financiera $y$ acceso a la vivienda". Disertación de Maestría por el Instituto de Economía. Universidad Federal do Rio de Janeiro, 2011. del IBGE. 
Holzmann, R. y Jorgensen, S. "Social Risk Management: a new conceptual framework for social protection and beyond". Paper Series Human Development Network, Social Protection. World Bank, 2000.

Instituto Brasileiro de Geografia e Estatística (IBGE). Pesquisa Nacional por Amostra de Domicílios (PNAD). Varios años.

Institute of Applied Economic Research (IPEADATA). Datos sobre mercado laboral.

Lavinas, Lena. "Transferências de renda: o quase tudo do sistema de proteção social brasileiro". Arrecadação, de onde vem? Gastos Públicos, para onde vão? Sicsú, João (ed.). São Paulo. Boitempo Editorial, 2007: 51-68.
Lavinas, Lena. "Pobreza: métricas e evolução recente no Brasil e no Nordeste". Cadernos do Desenvolvimento 5 (7). Centro Internacional Celso Furtado, 2010: 126-148.

Lavinas, Lena; Ferraz, Camila y Veiga, Alinne. "A experiência brasileira do crédito consignado: quais seus beneficiários?". Mimeo. Forthcoming in 2012 at Economia e Sociedade, UNICAMP, 2010.

Ministério do Desenvolvimento Social. Fome Zero. Uma história brasileira. III volumes. Veiga Almeida, Adriana (org.). Brasília, 2010.

Fecha de ingreso: 29/11/2011 Fecha de aprobación: 10/02/2012 
